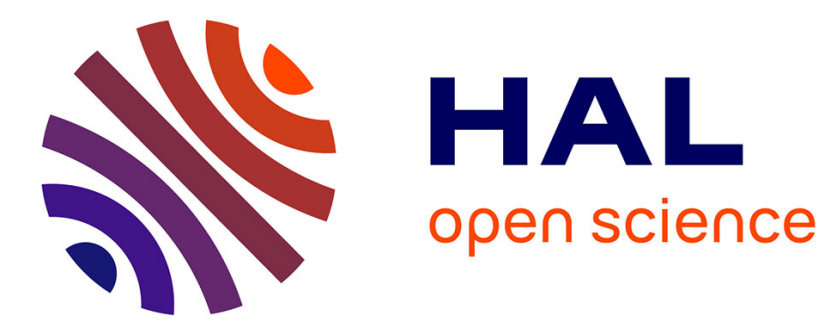

\title{
New techniques for simulating crystals
}

Martin Sweatman

\section{To cite this version:}

Martin Sweatman. New techniques for simulating crystals. Molecular Simulation, 2009, 35 (10-11), pp.897-909. 10.1080/08927020902769844 . hal-00515077

\section{HAL Id: hal-00515077 \\ https://hal.science/hal-00515077}

Submitted on 4 Sep 2010

HAL is a multi-disciplinary open access archive for the deposit and dissemination of scientific research documents, whether they are published or not. The documents may come from teaching and research institutions in France or abroad, or from public or private research centers.
L'archive ouverte pluridisciplinaire HAL, est destinée au dépôt et à la diffusion de documents scientifiques de niveau recherche, publiés ou non, émanant des établissements d'enseignement et de recherche français ou étrangers, des laboratoires publics ou privés. 


\section{Molecular Simulation}

Journal of

Experimental Nanoscience

- Taylor \& Francis

Taylor \& Francis Group

\section{New techniques for simulating crystals}

\begin{tabular}{|r|l|}
\hline Journal: & Molecular Simulation/Journal of Experimental Nanoscience \\
\hline Manuscript ID: & GMOS-2008-0244 \\
\hline Journal: & Molecular Simulation \\
\hline Date Submitted by the \\
Author: & $12-$ Dec-2008 \\
\hline Complete List of Authors: & $\begin{array}{l}\text { Sweatman, Martin; University of Strathclyde, Department of } \\
\text { Chemical and Process Engineering }\end{array}$ \\
\hline Keywords: & $\begin{array}{l}\text { crystals, free energy, Monte-carlo simulation, Gibbs simulation, } \\
\text { phase coexistence }\end{array}$ \\
\hline
\end{tabular}

\section{今scholarONE \\ Manuscript Central}




\title{
New techniques for simulating crystals
}

\author{
Martin B. Sweatman \\ Department of Chemical and Process Engineering, University of Strathclyde, \\ Glasgow, G1 1XJ
}

\begin{abstract}
Methods for simulating solid crystalline phases are generally not as straightforward as those for fluids. This work discusses the reason for this and reviews some recently developed Monte-Carlo techniques for simulating crystalline phases. The self-referential method for calculating crystal free energies is described first. This technique is particularly straightforward and it is expected to be very versatile. Next, a novel kind of Gibbs ensemble method adapted to treat crystalline solid - fluid coexistence is described. This technique requires free energy calculations of the crystalline phase as input, and of course these can be provided by the SR method.
\end{abstract}

\section{Introduction}

\subsection{Background}

The crystalline state is fundamental in nature, and molecular simulation is increasingly used to understand and predict the properties of matter. Yet, despite this, molecular simulation techniques for molecular crystals are in general not as satisfactory as those for fluids, and there is some debate about how confined crystals can be simulated correctly. This paper reviews some recent work that has made some progress in this area ${ }^{1-4}$ and which aims to make simulation of crystals, especially confined crystals, easier.

Crystal phase diagrams are usually much more complex than those of the corresponding fluid phases. This complexity increases when one considers molecular crystals, compared to atomic (spherical) species, and is likely to increase further under confinement, such as within a slit-pore, due to the 
shifting of bulk transitions and the potential introduction of surface induced transitions. The precise knowledge of a molecular crystal phase diagram can be of tremendous practical and economical interest, for instance in the pharmaceutical industry, where the impact of crystal polymorph can be almost as important as the choice of chemical compound on its possible prescription as a drug. And the prediction of freezing in confined environments, e.g. within porous materials, can be useful for many engineering processes that involve adsorption of fluids. Moreover, crystal phase diagrams can be sensitive to the choice of molecular model ${ }^{5}$, and so crystal phase diagram prediction can be used as another mechanism for fine-tuning molecular models. So it is important to be able to easily and straightforwardly map the phase diagrams of both bulk and confined molecular crystals via molecular simulation.

Central to the problem of simulating crystals is calculation of free energy. It is even more important to know the free energy for crystals than for fluids. This is not just because phase transitions involving crystalline solids are often strongly first-order and associated with significant hysteresis - a particular problem with simulations. It is also because we cannot impose or measure the bulk (i.e. experimental) pressure when simulating confined crystals, unless an impractically large system is simulated that includes the confined system - bulk system interface. Instead, it is essential that the chemical potential of the confined crystal is known (imposed or measured), because this quantity is the same in the confined and bulk systems at equilibrium. Unfortunately, we cannot impose the chemical potential on simulated crystals by performing grand-canonical ensemble simulations. The reasons for this are explained in detail in section 1.2 below. Instead, we should perform simulations that allow the crystal to relax to an equilibrium state, and then seek to measure the chemical potential of the crystal. For bulk crystals the isothermal-isobaric (NPT) ensemble ${ }^{6}$ is an obvious choice, while for crystals confined in uniform slit-pores, for example, simulations at constant interfacial tension $(\mathrm{N} \sigma \mathrm{T})$ are appropriate (Figure 1 shows a sketch of this ensemble). For a pure system the chemical potential is then simply the Gibbs free energy per particle. So for simulation studies of confined crystals calculation of the Gibbs free energy is essential if conditions inside the pore are to be related to experimental (or bulk) conditions, regardless of whether phase behaviour is of interest or not. In 
section 2 of this paper I summarize an approach for performing such free energy calculations, the selfreferential (SR) method, developed in recent years ${ }^{1-3}$, which is significantly more straightforward than other methods. Although the most recent version of this technique (which incorporates a kind of thermodynamic integration) has so far only been applied to bulk crystals, it is expected that it can also be applied unchanged to confined crystals.

The Gibbs ensemble $e^{6-8}$ is a convenient and popular Monte-Carlo method for simulating phase coexistence. Its main advantages are that coexisting states are generated spontaneously during a single simulation (given reasonable initial conditions) and that relatively small systems can be simulated because a phase interface is not required. Unfortunately, this method can fail if one or both of the coexisting phases are either very dense or solid. For dense liquid phases the method can fail if the acceptance rate for particle transfers between phases becomes too low, although specialised algorithms should improve transfer acceptance rates for certain cases ${ }^{9}$. However, the method fails when one or more of the phases are crystalline for a more fundamental reason, which is again discussed in section 1.2. However, it is possible to apply the Gibbs simulation technique to crystalline solid phases in an indirect way, and Section 3 summarizes work that shows how this can be achieved. This technique ${ }^{4}$ relies on generation of an accurate canonical Helmholtz free-energy model for the crystal phase. In turn, this free energy model relies on calculation of the Gibbs free energy for a trial crystalline state not too far from coexistence, which in turn can be calculated via the SR approach just discussed. So we see, once again, the importance of free energy calculations for simulations of crystalline solids.

This work summarizes both the SR method (section 2) and the Gibbs ensemble method applied to solid-fluid coexistence (section 3). But first in section 1.2 we discuss the reason why crystals are not as easy to simulate as fluids, namely because of conflict in symmetry requirements. 


\subsection{A conflict of symmetry requirements}

We know that for specified thermodynamic conditions (temperature and chemical potential or pressure) only one lattice site density corresponds to the equilibrium state. So, a simulated crystal must be able to adjust its lattice site density (provided it is not somehow initiated in the equilibrium state) in response to these external conditions. With grand-canonical simulations volume is fixed, so equilibrium is achieved by fluctuations in particle number in response to an applied chemical potential. However, when simulating a space-filling crystal with periodic boundary conditions this kind of fluctuation does not lead to a change in the lattice site density. Instead, it can lead only to changes in the number and kind of defects; it leads to the wrong kind of fluctuation. So in principle, regardless of the how well particle number fluctuations are sampled, equilibrium simulations of crystals cannot be performed with the grand canonical ensemble and periodic boundaries. Of course, for a space filling crystal fluctuations in lattice spacing in different directions can be achieved at fixed volume by shortening one box length while simultaneously lengthening another or by adjusting vertex angles. However, in this case spacing in one direction is traded against another, and although the crystal might be able to relax somewhat, this will still not allow full relaxation to the equilibrium lattice site density which is fixed because the overall volume and number of lattice sites is fixed. One might argue that it might be possible to develop Monte-Carlo moves that allow an entire slice of unit cells to be added or removed from one side of the crystal simulation box, or to adapt Tilwani and $\mathrm{Wu}^{10}{ }^{10}$ re-tiling algorithm to 3 -D crystals. But if this is achieved then in general this would not correspond to the grand canonical ensemble which requires molecule-by-molecule fluctuations rather than fluctuations in the number of unit cells. This problem is a matter of principle, and not concerned with clever insertion/deletion strategies.

Let us consider why this is the case. If instead the system was fluid, or even if it was a liquid crystal, i.e. if it did not possess crystalline translational symmetry in at least one direction, then the right kind of fluctuations could occur, even with periodic boundaries, in response to an applied chemical potential. So the fluid could adjust its average density perfectly in response to any change in chemical 
The same problem would arise if one attempted a standard Gibbs simulation involving a crystalline solid phase. Once again, the crystalline phase must be able to adjust its average lattice site density so that the chemical potentials of the coexisting phases are equilibrated. Although the lattice spacing can adjust so that pressure is equilibrated, it cannot adjust in response to particle exchanges which are responsible for equilibrating chemical potential. Any particle exchanges that do occur must result in creation or annihilation of defects, which by themselves do not create any change in the average lattice spacing.

Because of these problems it is standard practice to perform simulations of crystals with periodic boundary conditions using the isothermal-isobaric ensemble, or the equivalent ensemble for confined crystals where the interfacial tension (the negative of the average of the transverse component of the pressure tensor for a crystal confined within slit-pore for example) is fixed. Although the number of particles is fixed, the simulation cell volume can fluctuate with these simulations, and so the lattice site density can relax to its equilibrium value. 
Despite this, there are a rapidly growing number of examples ${ }^{11-33}$ in the literature where the grand canonical ensemble is used to simulate space-filling crystalline solids, particularly confined crystals. All this work should be considered carefully, because in every case these are not equilibrium simulations and each result is subject to systematic error. So the precise location of fluid-crystal phase transitions for bulk and confined systems are in doubt. In particular, Gubbins and colleagues ${ }^{12-33}$ have performed many studies of bulk and confined crystals using the grand canonical ensemble together with a kind of Landau free energy technique, which is essentially a non-Boltzmann or biased sampling method. The aim in these studies is to measure the (grand) free energy difference between a liquid and crystalline state. This is achieved by starting with one state (liquid or crystal) and traversing a path, along an order parameter that measures the system's degree of crystalline order, to the other state. The free energy difference is integrated along this path. The problem with this technique in this case is that the crystal end point of this path does not correspond to an equilibrium crystal at the specified chemical potential for the reasons given above, i.e. the volume is fixed in advance. If the simulation cell volume is large and the process is initiated with a liquid state, then as the path towards the crystal state is taken the system might adopt a lattice site density close to the equilibrium value. But this is not guaranteed, and the difference between the final lattice site density and the actual equilibrium lattice site density corresponding to the applied chemical potential will depend on the initial choice of volume. Despite this problem, results obtained using this method are in broad agreement with experiment, so it is likely that systematic are not large. Nevertheless, it is always preferable to formulate simulation strategies that are in principle exact so that the only error is statistical.

The above arguments also apply to quasi 2-dimensional crystals that form on the inner surface of a slit-pore where the pore width is fixed and fluid fills the middle of the pore. Once again, they key issue is whether the crystal can adjust its lattice site density to reach equilibrium in response to an applied chemical potential. If the pore width is fixed then the lattice site density is determined by the area of the slit-pore, which is fixed in advance with a grand canonical simulation. So simulations of crystals on the surface of slit-pores with fixed pore width using the grand canonical ensemble are also 


\section{The self-referential method}

As explained above in the introduction, methods for calculating the free energy are important for crystals, particularly confined crystals for which the chemical potential must be calculated if the corresponding bulk or reservoir state is to be determined. Several methods ${ }^{34-42}$ have been developed for calculating the canonical Helmholtz free energy of crystals, which are also sufficient for calculating the chemical potential provided the pressure is known or can be calculated (either the bulk pressure or the interfacial tension for a confined crystal). Probably the most popular such free energy methods are those based on an Einstein crystal reference state ${ }^{34,37,38,40-42}$, for which comprehensive texts $^{5,6,43,44}$ have been published.

However, the Einstein crystal route is not a very convenient method for confined crystals because the pore walls need to be 'integrated away' to make a path from the confined crystal to the Einstein crystal (the Einstein crystal is a non-interacting state, and so there cannot be any pore walls in this state). Additional simulations are needed to achieve this, and it is not clear that this is a straightforward procedure. For example, the precise path chosen might depend on the molecule - wall interaction type, i.e. hard or continuous potentials, and on the possible existence of any surface induced phase transitions. The aim of work that lead to the self-referential method ${ }^{3}$ (at least, the version of the self-referential method described here) was to develop a method that is able to treat bulk and confined crystals on an equal footing. That is, to develop a method that did not require the pore walls to be integrated away. So far, the version of the self-referential method described in the next section has been applied successfully only to bulk crystals ${ }^{1-3}$. Work aimed at applying it to 
confined crystals is ongoing, although it should be noted that the original version ${ }^{45}$ of the selfreferential method was applied in the context of hard rods on a line, which can be considered an extreme case of confinement for hard spheres in a cylindrical pore.

The self-referential (SR) method calculates the free energy difference between two crystalline solid systems that are identical except for their size (see Figure 2). If the free energy corresponding to the ensemble used is extensive, and if the large system is twice the size of the small system, then this free energy difference is also the free energy of the small system. So the small system is a kind of reference state, hence the name of this method. In early work Barnes and Kofke ${ }^{45}$ developed and applied the self-referential method to 1-D hard-rods on a line using the canonical ensemble and a technique based on sampling vibrational modes. They obtained good agreement with exact grand canonical results indicating that their system was sufficiently large. They coined the phrase 'selfreferential' for this general idea. Later, this idea was re-invented ${ }^{3}$ in the context of 3-dimensional crystals and applied to hard sphere and Lennard-Jones crystals using the isothermal-isobaric ensemble and a kind of 'parameter hopping' Monte-Carlo technique. Once again, comparison with reference literature results showed that the self-referential method was feasible and correct. However, this early version of the method is not efficient. The reason for this is that the free energy difference between the large and small system is actually relatively large, and so many parameter hops, typically several thousand - each requiring a single simulation, are needed to traverse the path between the large and small systems. Note that this particular technique is not an essential part of the SR method. Recent work $^{1,2}$ has shown how the efficiency of the SR method can be improved dramatically by implementing a kind of thermodynamic integration (SR-TI), to the point where the SR method can now be considered useful, and it is this version of the method that is summarized next. The latest version $^{1}$ also employs the canonical ensemble, which is considered slightly more convenient for bulk crystals, and has been extended and applied to crystals composed of rigid linear molecules (specifically homonuclear hard and Lennard-Jones dumbbells), and validated once more against reference literature data. This latest work also stated (but did not prove) that the method could be 


\subsubsection{Replication}

The replication step can be calculated analytically. To see this we first have to define the partition functions for the small, constrained double-size, and relaxed double-size systems. If we focus on particles with only positional coordinates initially for convenience, a canonical ensemble partition function for a system of $N$ such molecules is in general given by

$$
\Phi=\frac{\Lambda_{t}^{3 N}}{N !} \int_{V} d \mathbf{r}^{N} e^{-\beta H\left(\mathbf{r}^{N}, V\right)}
$$


where $r_{i}$ is the position of particle $i, d \mathbf{r}^{N}$ indicates the volume element corresponding to the $3 N$ positional coordinates of all the particles, $\Lambda_{t}=\sqrt{2 \pi m k_{B} T / h^{2}}$ is the translational contribution obtained by integrating over momenta, $H$ is the configurational contribution to the Hamiltonian and $\beta^{-1}=k_{B} T$ ( $k_{B}$ is Boltzmann's constant and $T$ is temperature). Note that the momentum contribution to the free energy is not important because it is the same for all phases, so it is dropped from here on. Only the configuration contribution is important in the context of phase behaviour. More complex particles with other degrees of freedom will be considered later.

Of course, we know that identical microstates must only be counted once, and this issue needs careful consideration $^{5,46,47}$. The factor of $N$ ! in (1) arises because normally there are no restrictions on the positions of molecules relative to each other. So each microstate has $N$ ! permutations, obtained by repeatedly swapping labels on pairs of molecules. However, if with a simulated crystal we restrict the positions of each molecule relative to each other, then only $N$ such permutations are possible. These $N$ permutations are obtained by translating the entire system (remember we have periodic boundary conditions), with fixed relative molecular positions, so that molecule 1 visits the position of each of the other lattice sites. So, provided our simulations only sample one permutation of relative molecular positions (this constraint is indicated by the symbol $V^{\prime}$ ), the partition function can be written as (without the momentum contributions now)

$$
\Phi=\frac{1}{N} \int_{V^{\prime}} d \mathbf{r}^{N} e^{-\beta H\left(\mathbf{r}^{N}, V\right)}
$$

Finally, by clamping molecule 1 at a particular position we can write this as

$$
\Phi=\frac{V}{N} \int_{V^{\prime}} d \mathbf{r}^{N-1} e^{-\beta H\left(\mathbf{r}^{N}, V\right)}
$$


Our aim, then, is to calculate the sum of the replication and relaxation free energy differences which are

$$
\beta \Delta(F)=\Delta\left(\beta F_{r e p}\right)+\Delta\left(\beta F_{r e l}\right)=-\ln \left(\frac{\Phi_{\alpha_{1}}}{\Phi_{s}}\right)-\ln \left(\frac{\Phi_{d}}{\Phi_{\alpha_{1}}}\right)
$$

where the subscripts stand for the single-size system $(s)$, the highly constrained self-similar doublesize system $\left(\alpha_{1}\right)$, and the normal double-size system $(d)$. This subscript notation is used throughout. Self-similarity of the double-size system is enforced via a constraint term in the configurational part of the Hamiltonian

$$
H_{\alpha}\left(r^{N_{d}}, V_{d}\right)=H_{0}\left(r^{N_{d}}, V_{d}\right)+\sum_{i=1}^{N_{s}} \phi\left(r_{i}-\alpha\right)
$$

where $r_{i}=\left|\mathbf{r}_{i+N_{s}}-\mathbf{L}_{x}-\mathbf{r}_{i}\right|, H_{0}$ is the Hamiltonian for an unconstrained system of particles, and where the constraint function is the infinite step-function,

$$
\phi(r)= \begin{cases}\infty ; & r>0 \\ 0 & ; \quad r \leq 0\end{cases}
$$

Note that the nature of the interaction between the particles has not been specified. It could be defined by a pair potential, or even a higher order multi-body potential. At present the only requirement is that the particles only have positional degrees of freedom, and so any pair potential is necessarily spherically symmetric. In equation (5) $N_{s}$ is the number of particles in the small system, $\mathbf{L}_{\mathbf{x}}$ is the 'replication vector' equal to half the double-size simulation box side (assumed to be in the x-direction here) and $\alpha$ controls the degree of self-similarity, or equivalently the degree of relaxation. That is, when $\alpha$ is very small the double-size system is almost perfectly self-similar and when $\alpha$ is very large 
the double-size system is perfectly relaxed. Indeed, when $\alpha=\alpha_{1}$ the self-similarity constraint is so severe that we can perform the integrals over the replicated particles degrees of freedom immediately because the Hamiltonian is essentially constant over the phase space accessible to each replicated particle. We obtain a contribution $V_{\alpha_{1}}=4 \pi \alpha_{1}^{3} / 3$ per replicated particle, i.e. the phase space volume available to each replicated particle. At the same time, because the double-size system is self-similar, we can write $H_{\alpha_{1}}\left(r^{N_{d}}, V_{d}\right) \cong 2 H_{0}\left(r^{N_{s}}, V_{s}\right)$, and so the replication term is

$$
\Delta\left(\beta F_{r e p}\right) \cong-\ln \left(\frac{\frac{V_{\alpha_{1}}^{N_{s}} V_{d}}{N_{d}} \int_{V_{s}^{\prime}} d \mathbf{r}^{N_{s}-1} \exp \left(-2 \beta_{\alpha_{1}} H_{0}\left(N_{s}, V_{s}\right)\right)}{\frac{V_{s}}{N_{s}} \int_{V_{s}^{\prime}} d \mathbf{r}^{N_{s}-1} \exp \left(-\beta_{s} H_{0}\left(N_{s}, V_{s}\right)\right)}\right)
$$

Notice that the temperature of the double-size system when $\alpha=\alpha_{1}$, denoted $\beta_{\alpha 1}$, is allowed to be different to the temperature of the system we are interested in, $\beta_{s}$. By setting $2 \beta_{\alpha_{1}}=\beta_{s}$ we obtain

$$
\Delta\left(\beta F_{r e p}\right) \cong-N_{s} \ln \left(V_{\alpha_{1}}\right)
$$

which is exact in the limit $\alpha_{1} \rightarrow 0$.

\subsubsection{Relaxation}

The relaxation free energy difference is conveniently calculated using a kind of thermodynamic integration

$$
\Delta\left(\beta F_{r e l}\right)=\int_{\alpha_{1}}^{\alpha_{m}} d \alpha \frac{d\left(\beta_{\alpha} F_{\alpha}\right)}{d \alpha}
$$




$$
\frac{d\left(\beta_{\alpha} F_{\alpha}\right)}{d \alpha}=-\frac{1}{\Phi_{\alpha}} \frac{d \Phi_{\alpha}}{d \alpha}
$$

The partition function depends on $\alpha$ through both the temperature and the Hamiltonian as follows. As $\alpha$ increases the temperature must decrease so that $\beta_{\alpha}=\beta_{s}$ when $\alpha=\alpha_{m}$. Also, as $\alpha$ increases the selfsimilarity constraint relaxes until it no longer has any effect. By the chain rule we obtain

$$
\frac{d\left(\beta_{\alpha} F_{\alpha}\right)}{d \alpha}=\left\langle\beta_{\alpha} \frac{\partial H_{\alpha}}{\partial \alpha}+H_{\alpha} \frac{\partial \beta_{\alpha}}{\partial \alpha}\right\rangle
$$

where $H_{\alpha}$ is the Hamiltonian corresponding to $\Phi_{\alpha}$ and hence $F_{\alpha}$, and where the angle brackets denote an ensemble average. So we have two contributions to $\Delta\left(\beta F_{\text {rel }}\right)$; one due to changes in temperature

$$
\Delta\left(\beta F_{T}\right)=\int_{\beta_{s} / 2}^{\beta_{s}}\left\langle H_{\alpha}\right\rangle d \beta_{\alpha}
$$

and another due to changes in the self-similarity constraint,

$$
\Delta\left(\beta F_{\alpha}\right)=\int_{\alpha_{1}}^{\alpha_{m}} d \alpha\left\langle\beta_{\alpha}\left(\frac{\partial H_{\alpha}}{\partial \alpha}\right)\right\rangle_{\alpha}
$$

The first contribution, $\Delta\left(\beta F_{T}\right)$, can be evaluated using numerical quadrature. To calculate contribution (13) we must first differentiate the Hamiltonian with respect to $\alpha$. This is not so straightforward, and rather than repeating the derivation in earlier work the result is simply stated 
$\Delta\left(\beta F_{\alpha}\right)=-4 \pi N_{s} \int_{\alpha_{1}}^{\alpha_{m}} d \alpha g_{\alpha}(r=\alpha) \alpha^{2}$

Here, $g_{\alpha}(r)$ is the average 'partner' distribution function $g_{\alpha}(r)=N_{s}^{-1} \sum_{i} g_{\alpha i}\left(r_{i}=r\right)$, where $g_{\alpha i}\left(r_{i}\right)$ is the individual partner distribution function for the separation $r_{i}=\left|\mathbf{r}_{i+N_{s}}-\mathbf{r}_{i}-\mathbf{L}_{x}\right|$ of any particle replicated particle pair when the self-similarity constraint is $\alpha$ and the temperature is $\beta_{\alpha}$. In (14) this distribution function is evaluated at its 'edge' when $r=\alpha$.

However, in the limit $\alpha_{1} \rightarrow 0$, we find that $4 \pi g_{\alpha}(r) \alpha^{2} / 3 \cong 1 / \alpha$, and so greater numerical accuracy is achieved by integrating with respect to $\ln (\alpha)$. This transforms (14) to

$$
\Delta\left(\beta F_{\alpha}\right)=-4 \pi N_{s} \int_{\ln \left(\alpha_{1}\right)}^{\ln \left(\alpha_{m}\right)} d \ln (\alpha) g_{\alpha}(r=\alpha) \alpha^{3}
$$

Putting (8), (12), and (15) together gives our final result for the configurational contribution, which in terms of a length scale $\lambda$ is

$$
\frac{\Delta(\beta F)}{N_{s}} \cong-\ln \left(\frac{V_{\alpha_{1}}}{\lambda^{3}}\right)+\frac{1}{N_{s}} \int_{\beta_{s} / 2}^{\beta_{s}} d \beta\left\langle H_{\alpha}\right\rangle-4 \pi \int_{\ln \left(\alpha_{1}\right)}^{\ln \left(\alpha_{m}\right)} d \ln (\alpha) g_{\alpha}(r=\alpha) \alpha^{3}
$$

which is exact in the limits $\alpha_{1} \rightarrow 0$ and $\alpha_{m} \rightarrow \infty$. Obviously, for computer calculations finite limits need to be chosen for $\ln \left(\alpha_{1}\right)$ and $\ln \left(\alpha_{m}\right)$. For the upper limit, $\alpha_{m}$ is large enough when $g_{\alpha_{m}}\left(r=\alpha_{m}\right) \sim 0$. For a crystal there will always exist $\alpha_{m}<l$ for which this occurs, where $l$ is the maximum lattice spacing in any direction. On the other hand, in general it is not known in advance how small $\alpha_{1}$ needs to be for a desired level of accuracy. However, it is known that in the limit 


\subsection{Rigid linear molecules}

To calculate the integrals in (16) a series of simulations for a range of values of $\alpha$ must be performed. Each simulation is essentially an ordinary canonical ensemble Monte-Carlo simulation of the doublesize system, except that the constraint on the configurational Hamiltonian defined in (5), which depends on $\alpha$, must be included. It is important to perform compound MC moves to ensure efficient sampling when $\alpha$ is small. These moves are described in the earlier work ${ }^{1-3}$ in detail, but briefly they consist of simultaneous trial moves for both particle $i$ and its partner, $i+N_{s}$. A trial move consists of displacing both particles by the same (large) amount, and then displacing one of them a (small) amount more. Recall particle 1 is clamped and so is never displaced. Also, the temperature path from $\beta_{s} / 2$ to $\beta_{s}$ needs to be defined. In fact this path will depend on $\alpha$. In paper 1 a suitable path is found to be one that keeps the total average configurational energy, $\left\langle H_{\alpha}\right\rangle$, nearly constant as $\alpha$ changes. An algorithm for choosing $\beta_{\alpha}$ that achieves this is defined in that work, although it is not unique and many other algorithms can be defined. This kind of path, i.e. one that keeps the configurational energy nearly constant, is thought to work well because it is important to avoid any potential phase transitions. A phase transition could occur if the pressure of the double-size constrained system for any particular value of $\alpha$ changes substantially from that of the unconstrained system. And this can happen if the average energy of the double-size constrained system changes substantially from that of the unconstrained system. So a temperature path that keeps the average configurational energy nearly constant appears to be suitable. 
Equation (16) is valid only for spherical particles, which include hard spheres and Lennard-Jones particles. In paper 1 it was shown how the SR method can be extended to rigid linear molecules, such as needles and dumbbell shaped molecules, and results obtained for hard and Lennard-Jones dumbbells compared well with reference data in the literature ${ }^{48-50}$. To make the extension to rigid linear molecules we first have to consider and describe the degrees of freedom of such a molecule. In addition to the position of the molecular centre, the direction of a rigid linear molecule can be described in terms of Euler angles, i.e. $\theta$ describes its polar angle (the angle between a molecule's director and the z-Cartesian axis) and $\phi$ describes its azimuthal angle (the angle formed by the projection of the director onto the $\mathrm{x}$-y plane and the $\mathrm{x}$-axis). The $z$-axis and $\mathrm{x}$-axis referred to here are fixed in space. However, when using the SR method it is convenient to express the orientational coordinates of each replicated molecule with respect to the coordinates of that molecules original partner molecule. So, for each replicated molecule $\tau$ is the angle between the directors of a replicated molecule and its partner, while $\varphi$ is the azimuthal angle of a replicated particle with respect to coordinates fixed to the director of the original particle. Using this convention, which is described in detail in terms of rotation matrices in paper 1, the Hamiltonian for the constrained double-size system can now be written as

$$
H_{\alpha}\left(r^{N_{d}}, V_{d}\right)=H_{0}\left(r^{N_{d}}, V_{d}\right)+\sum_{i=1}^{N_{s}} \phi\left(r_{i}-\alpha\right)+\sum_{i=1}^{N_{s}} \phi\left(\eta_{i}-\hat{\eta}_{\alpha}\right)
$$

where $\eta_{i}=1-\cos \left(\tau_{i}\right)$ and $\hat{\eta}_{\alpha}=\hat{\eta}(\alpha)$ is the constraint on each $\eta_{i}$. Recall, this constraint is effectively on the angle $\tau_{i}$ between the directors of each replicated particle and its original partner. So each replicated particle's director can point anywhere within a circle on the surface the unit sphere centred on the director of the original particle. These constraints are illustrated in Figure 4. Using this convention it is once more straightforward to derive the corresponding replication and relaxation terms following the same procedure as for positional degrees of freedom. The corresponding free energy expression for rigid linear molecules is 
The upper limit, $\hat{\eta}_{m}$, depends on the kind of particle and crystal phase being studied. For molecules with an inversion centre (e.g. homonuclear dumbbells) the maximum value for $\hat{\eta}_{m}$ is 1 , and for plastic crystals (these are crystals without long-range orientational order) it is important to use this maximum limit. However, for orientationally ordered crystals of these molecules $\hat{\eta}_{m}$ is large enough when $g_{\hat{\eta}_{m}}\left(\eta=\hat{\eta}_{m}\right) \sim 0$. On the other hand, for plastic crystals composed of molecules without an inversion centre (e.g. heteronuclear dumbbells) it is important to set $\hat{\eta}_{m}=2$. Finally, for orientationally ordered crystals composed of these molecules $\hat{\eta}_{m}$ is again large enough when $g_{\hat{\eta}_{m}}\left(\eta=\hat{\eta}_{m}\right) \sim 0$. However, one also has to ensure that all the possible permutations for 'up' and 'down' molecules are properly sampled. This is similar to the problem of residual entropy for water ice ${ }^{5}$. If we now consider $\hat{\eta}_{1}$, it is small enough when $g_{\hat{\eta}_{1}}\left(\eta=\hat{\eta}_{1}\right) \hat{\eta}_{1} \sim 1$. Clearly, the functional form for $\hat{\eta}(\alpha)$ should be chosen to reproduce these limits, and in paper 1 a simple linear relationship is found to be sufficient. Figure 5 shows how $g_{\hat{\eta}_{\alpha}}\left(\eta=\hat{\eta}_{\alpha}\right) \hat{\eta}_{\alpha}$ depends on $\hat{\eta}_{\alpha}$ for an $\alpha-\mathrm{N}_{2}$ face-centred-cubic ${ }^{1}$ crystal of hard dumbbells with reduced bond length $L^{*}=L / \sigma=0.3$ and reduced molecular density $\rho^{*}=\rho \sigma^{3}=0.883397$, where $\sigma$ is the diameter of one site of the dumbbell. 
In paper 1 the (configurational) canonical Helmholtz free energy difference (18) for some facecentred-cubic crystals composed of hard and Lennard-Jones dumbbells was evaluated for several different systems, including some orientationally ordered and plastic crystals, and in general the results compared well with values for the absolute canonical Helmholtz free energy obtained using an Einstein crystal (Frenkel-Ladd) based approach ${ }^{48-50}$. Serious disagreement between the SR and FL approaches was found only for one of the LJ dumbbell systems.

\subsection{Extension to other molecules and ensembles}

We see by comparing (16) and (18) how a general expression for the free energy of arbitrary spacefilling crystals can be developed. There is a contribution to the replication term from each degree of freedom corresponding to the phase space volume available to a replicated particle when the system is most constrained at $\alpha=\alpha_{1}$. For rigid molecules there is a contribution due to the translational degrees of freedom of the molecular centre $\left(V_{\alpha_{1}}=4 \pi \alpha_{1}^{3} / 3\right)$. For rigid linear molecules there is an additional contribution due to the solid angle described by a molecule's director which, because only the relative polar angle needs to be constrained for these molecules (since the Hamiltonian is essentially independent of the relative azimuthal angle when the polar angle is very small), is $V_{\hat{\eta}_{1}}=2 \pi \hat{\eta}_{1}$. For general rigid molecules without any internal symmetries we expect an additional contribution due to a constraint on internal molecular rotations about the major axis, described by $-\pi \leq \kappa \leq \pi$, equal to $V_{\hat{\kappa}_{1}}=2 \hat{\kappa}_{1}$, where $\hat{\kappa}_{1}$ is the initial constraint on $|\kappa|$. Once again, for the same reason given above, it should not be necessary to constrain the relative azimuthal angle, and so $V_{\hat{\eta}_{1}}$ remains equal to $2 \pi \hat{\eta}_{1}$. For non-rigid molecules there is some freedom in the description of the molecular degrees of freedom. For example, for a non-rigid homonuclear dumbbell molecule one could choose to describe a molecule either in terms of the position of the molecular centre, the orientation of the molecular director and the bond length, or alternatively in terms of the positions of the two sites. Because with the SR method it is convenient to express the coordinates of each replicated particle relative to those 
of each corresponding original particle, a replicated particle could be described in terms of either $r_{i}$, $\eta_{I}, \phi_{i}$ and the difference in bond length, or alternatively in terms of the positions of the two sites relative to those of the original molecule. Each choice will lead to different expressions for both the replication and relaxation terms, but of course the free energies calculated by either choice should be the same.

In general, we can expect the replication term for a pure crystal to be given by the analytic expression

$$
\Delta\left(\beta F_{\text {rep }}\right)=-N_{s} \ln \left(\frac{\Psi_{1}}{\lambda^{d}}\right)
$$

where $\Psi=\prod_{j} f_{j}\left(\hat{r}_{j}\right)$ is the phase-space available to a single particle (which is just the product of the phase space functions $f_{j}\left(\hat{r}_{j}\right)$ for each degree of freedom $r_{j}$, while $\Psi_{1}=\prod_{j} f_{j}\left(\hat{r}_{j 1}\right)$ is the phasespace volume available to each replicated particle when the system is fully constrained, and $d$ is the dimensionality of the crystal.

By comparing (16) and (18) we see that for each degree of freedom relaxed during the relaxation step that there is an integral over its respective partner distribution function. Each corresponding integrand is multiplied by the rate of change of the phase space volume, so we expect the replication term can be generalised to

$$
\Delta\left(\beta F_{r e l}\right)=\int_{\beta_{s} / 2}^{\beta_{s}} d \beta_{\alpha}\left\langle H_{\alpha}\right\rangle-\left.\sum_{j^{\prime}} \int_{\ln \left(\hat{r}_{j 1}\right)}^{\ln \left(\hat{r}_{j m}\right)} d \ln \left(\hat{r}_{j \alpha}\right) g_{\hat{r}_{j \alpha}}\left(r_{j}=\hat{r}_{j \alpha}\right) \hat{r}_{j \alpha} \frac{d \Psi}{d \hat{r}_{j}}\right|_{\hat{r}_{j \alpha}}
$$


where the sum need only be over those degrees of freedom that were constrained upon replication, indicated by $j^{\prime}$, and hence need to be relaxed. In addition, as with water ice, any kind of residual entropy would need to be accounted for.

The sum of (19) and (20) gives a rather compact and simple expression for the Helmholtz free energy of crystals

$$
\frac{\Delta(\beta F)}{N_{s}}=-\ln \left(\frac{\Psi_{1}}{\lambda^{d}}\right)+\int_{\beta_{s} / 2}^{\beta_{s}} d \beta_{\alpha}\left\langle H_{\alpha}\right\rangle-\left.\sum_{j^{\prime}}^{\ln \left(\hat{r}_{j m}\right)} \int_{\ln \left(\hat{r}_{j 1}\right)} d \ln \left(\hat{r}_{j \alpha}\right) g_{\hat{r}_{j \alpha}}\left(r_{j}=\hat{r}_{j \alpha}\right) \hat{r}_{j \alpha} \frac{d \Psi}{d \hat{r}_{j}}\right|_{\hat{r}_{j \alpha}}
$$

Because the replication term is analytic only one kind of simulation is needed that measures the respective partner distribution functions at their 'edges', $g_{\hat{r} j \alpha}\left(r_{j}=\hat{r}_{j \alpha}\right)$, and the average configurational energy, $\left\langle H_{\alpha}\right\rangle$, for a suitable range of values of the relaxation parameter $\alpha$, and for a suitable $\beta$-path. The corresponding generalised Hamiltonian for these constrained double-size simulations can be defined as

$$
H_{\alpha}\left(\{\mathbf{r}\}^{N_{d}}, V_{d}\right)=H_{0}\left(\{\mathbf{r}\}^{N_{d}}, V_{d}\right)+\sum_{j^{\prime}} \sum_{i=1}^{N_{s}} \phi\left(r_{i j}-\hat{r}_{j \alpha}\right)
$$

where $\{\mathbf{r}\}^{N_{d}}$ indicates all particle coordinates (positions, orientations etc.), and $r_{i j}$ is the $j$ th constrained degree of freedom, corresponding to the constraint $\hat{r}_{j \alpha}$, which depends on the difference between the coordinates of an original particle $i$ and its replicated partner $i+N_{s}$.

At no point have any assumptions about the nature of molecule - molecule interactions or the presence of an external field been made (except that any external field should have the same translational symmetry as the single-size simulation cell in the replication direction), and so, without 
any change in the technique, it is expected that the SR method can be applied to systems involving pairwise or higher body interactions and to systems involving external fields, such as pore walls, provided they have the required translational symmetry. Clearly, further work is needed to confirm these expectations.

Another attractive feature of the SR method is that the centre of mass is not constrained, and so no centre of mass corrections ${ }^{42}$ are needed. Also, it was shown in paper 2 that the SR method can be used with the isothermal-isobaric ensemble. For these simulations all quantities become ensemble averaged with respect to volume fluctuations. The reduced Gibbs free energy difference, $\Delta(\beta G)$ for spherical particles was obtained as

$$
\frac{\Delta(\beta G)}{N_{s}} \cong-\ln \left\langle\frac{V_{\alpha_{1}}}{\lambda^{3}}\right\rangle_{s}-\frac{\ln (2)}{N_{s}}+\frac{1}{N_{s}} \int_{\beta_{s} / 2}^{\beta_{s}} d \beta\left\langle P_{s} V_{d}+H\right\rangle_{\alpha}-4 \pi \int_{\ln \left(\alpha_{1}\right)}^{\ln \left(\alpha_{m}\right)} d \ln \alpha\left\{g_{\alpha}\left(r=\alpha L_{x}\right) \alpha^{3} L_{x}^{3}\right\}_{\alpha}
$$

Here, there are several new terms and some new notation that require explanation. But first, an important difference between this isothermal-isobaric ensemble derivation and the canonical ensemble derivation must be described. This difference concerns the Hamiltonian for the constrained double-size system, which for spherical particles is now written

$$
H_{\alpha}\left(r^{N_{d}}, V_{d}\right)=H_{0}\left(r^{N_{d}}, V_{d}\right)+\sum_{i=1}^{N_{s}} \phi\left(r_{i}-\alpha L_{x}\right)
$$

Note the factor $L_{x}$. Although important, it is actually quite trivial to implement this constraint and clearly it is possible to define the constrained Hamiltonian is this way for the canonical version as well. So this can be considered a generalised form of the self-similarity constraint. Inclusion of this factor in (24) allows the self-similarity constraint to fluctuate in tandem with the volume. This difference ensures that volume fluctuations are not biased by the self-similarity constraint (actually, this form of the Hamiltonian assumes that volume fluctuations occur via Monte-Carlo moves that 
uniformly scale the volume, which is acceptable provided the shape of the simulation cell is already consistent with the equilibrium shape of the crystal unit cell). Switching $\alpha$ for $\alpha L_{x}$ also results in $\alpha^{3}$ becoming $\alpha^{3} L_{x}^{3}$ in the right most term in (23), and in $V_{\alpha_{1}}$ becoming $4 \pi \alpha^{3} L_{x}^{3} / 3$. Also note the appearance of a $\ln (2)$ term and the term $P_{s} V_{d}$, where $P_{s}$ is the pressure and $V_{d}$ is the volume of the double-size system. The final difference is that each term on the right hand side of (23) is an ensemble average. The angle brackets denote an ensemble average with respect to all fluctuations. But because $g_{\alpha}$ is already an ensemble average with respect to particle positions, the curly brackets denote a further ensemble average of $g_{\alpha}$ only with respect to volume fluctuations.

Another important practical difference in evaluating (23) compared to (16) concerns how the $\beta$-path is defined during relaxation, that is how temperature is adjusted during relaxation. The canonical ensemble version employs an algorithm that attempts to keep the average (configurational) energy of the double-size system nearly constant during relaxation. As explained earlier, the rationale for this is that if the average energy is roughly constant during relaxation then the pressure might also be nearly constant, and so the crystal should not undergo any change of phase. However, with the isothermalisobaric version an algorithm can be designed that works directly with the volume. That is, in paper 2 an algorithm is employed that attempts to keep the average volume nearly constant during relaxation. It is not clear whether this choice for the $\beta$-path is important, or whether the nearly constant average energy algorithm would also be suitable for the isothermal-isobaric version.

Following the same arguments that lead to (21), the general expression for the Gibbs free energy difference is

$$
\frac{\Delta(\beta G)}{N_{s}} \cong-\ln \left\langle\frac{\Psi_{1}}{\lambda^{3}}\right\rangle_{s}-\frac{\ln (2)}{N_{s}}+\frac{1}{N_{s}} \int_{\beta_{s} / 2}^{\beta_{s}} d \beta\left\langle P_{s} V_{d}+H\right\rangle_{\alpha}-\sum_{j^{\prime}} \int_{\ln \left(\hat{r}_{j_{1}}\right)}^{\ln \left(\hat{r}_{j n}\right)} d \ln \hat{r}_{j}\left\{g_{\hat{r}_{j}}\left(r_{j}=\hat{r}_{j}\right) \hat{r}_{j} \frac{\partial \Psi}{\partial \hat{r}_{j}}\right\}_{\alpha}
$$


where for any positional degree of freedom we must let the self-similarity constraint fluctuate in tandem with the volume. Once again, this expression does not account for any residual entropy. Future work will aim to test this result.

\section{The Gibbs ensemble adapted to crystalline solid - fluid coexistence}

A fluid system with fixed volume at sub-critical temperature and density between the coexisting vapour and liquid densities will spontaneously phase separate to form the corresponding vapour and liquid phases because this macrostate has the lowest Helmholtz free energy. A state with even lower free energy is one without a vapour-liquid interface. While it is not possible to avoid the formation of an interface in nature under these circumstances, it is possible to do this in molecular simulations, and this is the basis of the NVT Gibbs ensemble simulation method ${ }^{6-8}$. The advantage this affords is that the uniform bulk phases at coexistence can be simulated, and this requires far fewer particles than a simulation with an interface if accurate bulk fluid properties are required. Since its invention this technique has become a very popular method ${ }^{51}$ for determining fluid-fluid coexistence. Unfortunately, for the reason outlined in section 1.2, it is, in principle, impossible to perform Gibbs ensemble simulations when one of the phases is a crystalline solid in a direct way. However, it is possible to perform these simulations in an indirect way, and this section summarizes work ${ }^{4}$ that demonstrates this.

Once again, we start by considering the configurational partition function of a pure system, this time for a 'two box' simulation where box 1 contains crystalline solid and box 2 contains fluid, both with periodic boundaries

$$
\Psi=\sum_{N_{1}=00}^{N} \int_{0}^{V} d V_{1} \int \frac{d \mathbf{r}^{N_{1}}}{N_{1} !} \exp \left(-\beta H\left(\mathbf{r}^{N_{1}}, V_{1}\right)\right) \int \frac{d \mathbf{r}^{N_{2}}}{N_{2} !} \exp \left(-\beta H\left(\mathbf{r}^{N_{2}}, V_{2}\right)\right)
$$

Here, $\mathbf{r}$ represents all molecular degrees of freedom, i.e. position, orientation etc., and for an NVT Gibbs simulation we have $V=V_{1}+V_{2}$ and $N=N_{1}+N_{2}$. The key idea of paper 4 is to replace the 
crystalline solid simulation box by a free energy model of the crystalline solid, i.e. to rewrite the partition function as

$\Psi=\sum_{N_{1}=0}^{N} \int_{0}^{V} d V_{1} \exp \left(-\beta F\left(N_{1}, V_{1}\right)\right) \int \frac{d \mathbf{r}^{N_{2}}}{N_{2} !} \exp \left(-\beta H\left(\mathbf{r}^{N_{2}}, V_{2}\right)\right)$

where $F\left(N_{1}, V_{1}\right)$ is the canonical Helmholtz free energy for a system with $N_{1}$ molecules in volume $V_{1}$. Provided $F$ is known accurately for all values of $N_{1}$ and $V_{1}$ that are sampled during a simulation, then ensemble averages can be calculated accurately for either phase, provided the fluid can be simulated in box 2. Mehta and Kofke ${ }^{52}$ used a similar idea in their application to vapour-liquid coexistence at low temperatures by employing a free energy model for a low density vapour phase in coexistence with a simulated liquid phase.

To make progress, a free energy model of the crystalline solid phase is required for all values of $N_{1}$ and $V_{1}$ likely to be sampled during a Gibbs simulation. Generally, a free energy model can be obtained as follows. First, a Taylor series expansion in inverse powers of the number of molecules, $\delta$ $=1 / N$, for the free energy per particle $f_{\rho}=F(N, N / \rho) / N$ at a particular density $\rho$, can be written

$f_{\rho}\left(\delta_{1}\right)=f_{\rho}(0)+\left.\delta_{1} \frac{\partial f_{\rho}}{\partial \delta}\right|_{\delta=0}+O\left(\delta_{1}^{2}\right)$

From this, it follows immediately that

$$
f_{\rho}\left(\delta_{1}\right) \approx f_{\rho}\left(\delta_{c}\right)+\left.\left(\delta_{1}-\delta_{c}\right) \frac{\partial f_{\rho}}{\partial \delta}\right|_{\delta=0}
$$


and hence the canonical Helmholtz free energy for a system with $N_{1}$ particles in volume $V_{1}$ can be written in terms of the canonical Helmholtz free energy of a system with $N_{c}$ molecules at the same density as

$$
F\left(N_{1}, V_{1}\right) \approx \frac{N_{1}}{N_{c}} F\left(N_{c}, V_{1} \frac{N_{c}}{N_{1}}\right)+\left.\left(1-\frac{N_{1}}{N_{c}}\right) \frac{\partial f_{\rho}}{\partial \delta}\right|_{\delta=0}
$$

which is accurate for sufficiently large $N_{1}$ and $N_{c}$. This analysis has simplified the problem from that of knowing the free energy for each combination of $N_{1}$ and $V_{1}$ to instead just knowing the free energy for a fixed number of molecules, $N_{c}$, in a range of volumes, and the constant $\left(\partial f_{\rho} / \partial \delta\right)_{\delta=0}$. In paper 2 equation (30) is truncated at its first term resulting in a simple linear scaling approximation for $F$

$$
F\left(N_{1}, V_{1}\right) \approx \frac{N_{1}}{N_{c}} F\left(N_{c}, V_{1} \frac{N_{c}}{N_{1}}\right)
$$

leaving just $F\left(N_{c}, V\right)$ to be determined for a range of values of $V$. This function can be found from the volume probability distribution function, $p(V)$, of an isothermal-isobaric (NPT) simulation with $N_{c}$ particles at pressure $P$

$$
F\left(N_{c}, V\right)=-k_{B} T \ln p(V)-P V+\mu N_{c}
$$

where $\mu$ is the chemical potential corresponding to this pressure (note the sign error in equation (16) in paper 4). To use (31) a pressure should be chosen such that the range of densities sampled during the NPT simulation is similar to that sampled during the Gibbs simulation. The chemical potential at this pressure must also be known, and this can be determined by any free energy method for crystalline solids, including the SR method described in the preceding sections. In paper 4, for convenience, a pressure for which i) the chemical potential was already known, and ii) it was also 
known that the appropriate range of densities would be sampled, was chosen. However, in general, an appropriate pressure is not usually known in advance, and so several iterations of this Gibbs simulation technique might be required to obtain convergent results. Future work will test this aspect of this method.

In paper 4 a free energy model for the face centred cubic phase of a crystalline (shifted force) Lennard-Jones system was generated by combining (31) and (32) to obtain

$$
F\left(N_{1}, V_{1}\right)=\frac{-k_{B} T N_{1}}{N_{c}} \ln p\left(V_{1} \frac{N_{c}}{N_{1}}\right)-P V_{1}+\mu N_{1}
$$

This free energy model, when combined with (27), enabled Gibbs ensemble simulations of both vapour - solid and liquid - solid coexistence at the triple point of this Lennard-Jones system. The known pressure and chemical potential at this point were used to generate the free energy for $N_{c}=256$ particles at this state. This data is shown in Figure 6, and is used as input to (31). Using this free energy model, good agreement was found for the density, pressure and chemical potential of the coexisting phases at the triple point between the indirect Gibbs simulations described here and from reference values in the literature ${ }^{53}$ (to within statistical error).

Clearly, this approach is not as convenient as standard Gibbs simulations for gas-liquid coexistence because usually a suitable pressure and chemical potential at which to generate the canonical Helmholtz free energy model via NPT simulation is not known in advance. In paper 4 a state point for which this data was known in advance was specifically chosen for convenience to test the method. But usually this information will not be known. Also, this method is less convenient than standard Gibbs simulations of vapour-liquid coexistence because several types of simulation are needed to generate the free energy model, including a free energy calculation for the crystalline solid. Future work will aim to compare this indirect Gibbs ensemble strategy with the usual alternative strategy, which is calculation of the free energy along both solid and fluid branches near coexistence ${ }^{5}$. Also 
note the work of Chen et.al. ${ }^{54}$ concerning simulation of fluid-solid coexistence. Their Gibbs simulation technique is designed to allow simulation of phase coexistence between liquid and solid phases. However, in their work equilibrium between the liquid phase and the solid phase is achieved via a liquid - solid interface. So, the title of their paper is not strictly accurate. Nevertheless, despite the fact that their method requires many more particles due to the presence of an interface, it could be quite convenient.

Despite the initial success of the indirect Gibbs simulation technique summarized above, several problems with this method should be highlighted. First, as with any Gibbs simulation, it becomes very slow if the liquid phase is dense because the probability of a successful particle exchange between boxes becomes very low. In this case specialized 'biasing' techniques ${ }^{9}$ might be useful. Second, if the density difference between coexisting phases is small, which is often the case for coexisting liquid and solid phases, then large fluctuations in the number of particles in each box should occur. In this case very long simulations are needed to ensure sufficient sampling of these large fluctuations. In addition, a more accurate approximation than (33) for the free energy model might be needed. This model relies on the simple linear scaling approximation (31), which is expected to loose accuracy when either $N_{c}$ or $N_{1}$ are small. A more sophisticated approach could use both the first and second terms in (30), with at least two NPT simulations performed at the same pressure, but with significantly different numbers of particles, to determine $\left(\partial f_{\rho} / \partial \delta\right)_{\delta=0}$. Finally, this approach has, so far, only been applied to pure crystals and it is not yet clear if crystalline mixtures can be simulated in this way. 


\section{Figures}

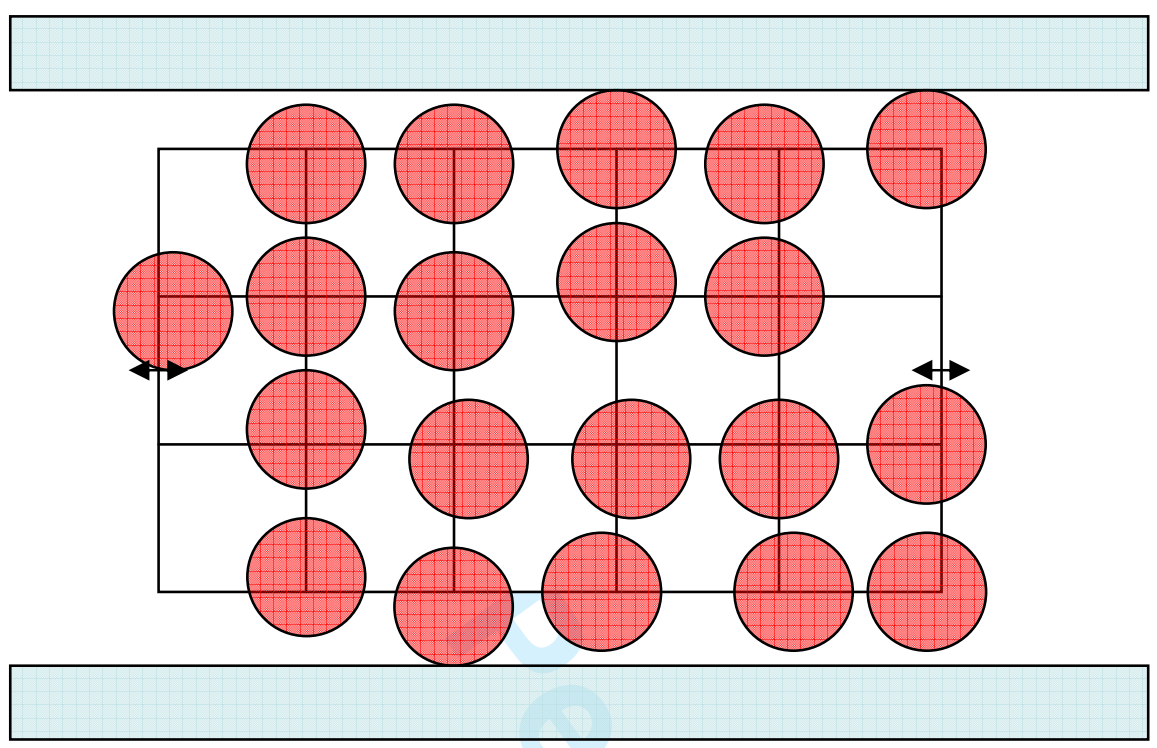

Figure 1. Sketch of the NoT ensemble for simulation of crystalline solid in a slit pore. Fluctuations in volume are allowed in the direction of the slit pore to equilibrate the interfacial tension, $\sigma$. Periodic boundaries are applied in directions parallel to the pore walls.

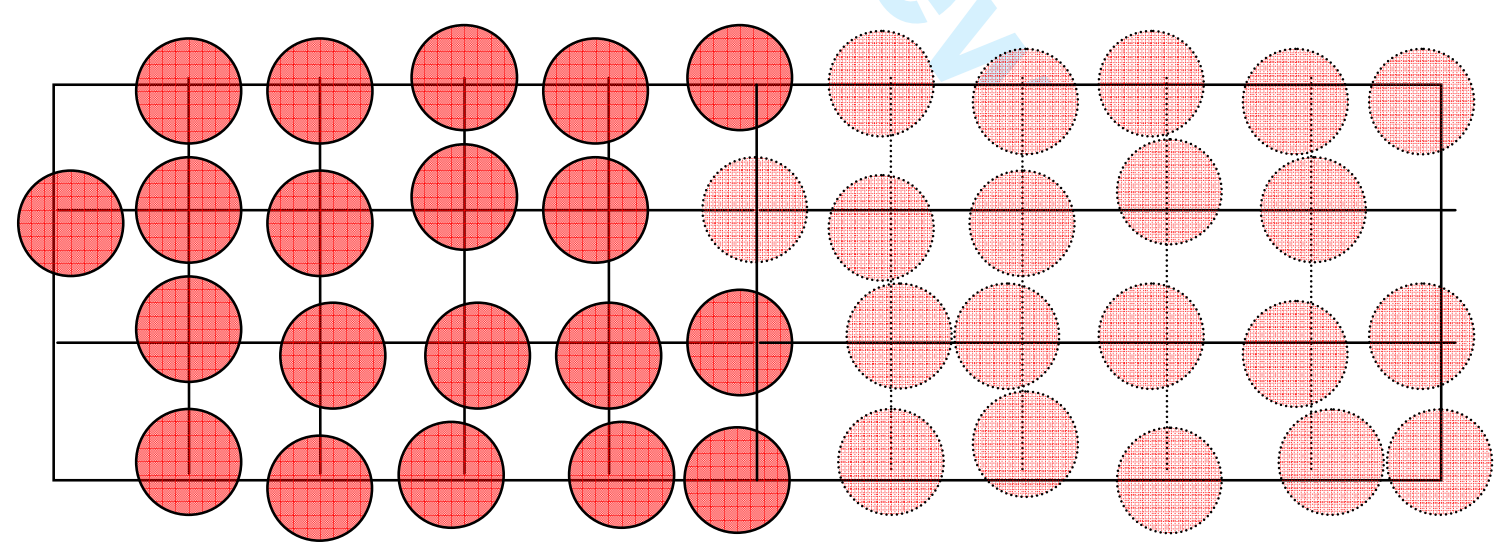

Figure 2. Sketch of the basic idea behind the self-referential method; the Gibbs free energy of the double-size system is twice that of a single-size system. Note that these are not isolated systems; periodic boundaries are applied. 


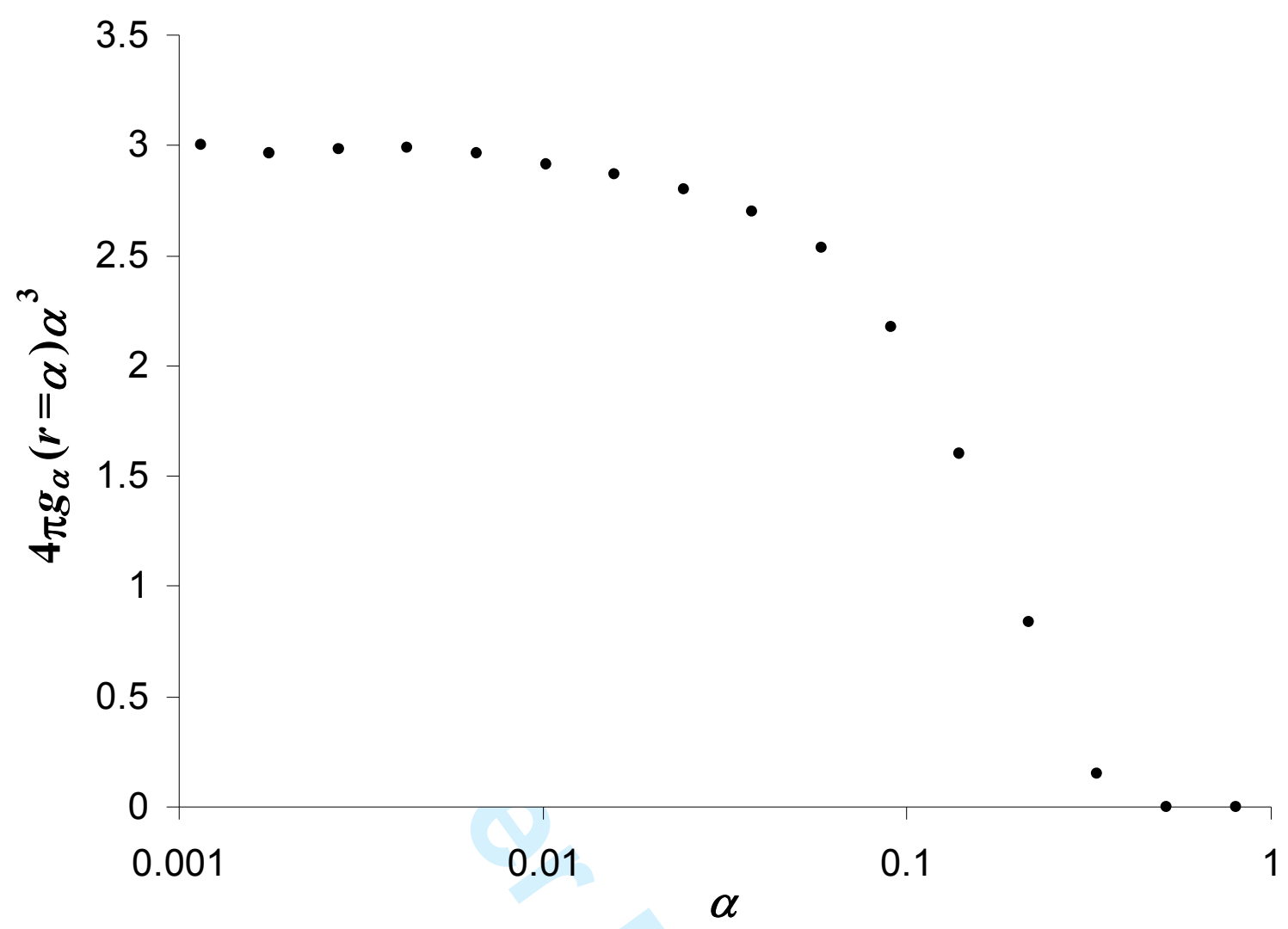

Figure 3. The variation of $4 \pi g_{\alpha}(r=\alpha) \alpha^{3}$ with $\alpha$ for a hard sphere fcc crystal with reduced density (average number of hard spheres per hard-sphere diameter cubed) 1.04086. Symbol sizes are larger than the standard error, and $\alpha$ is on a logarithmic scale. 


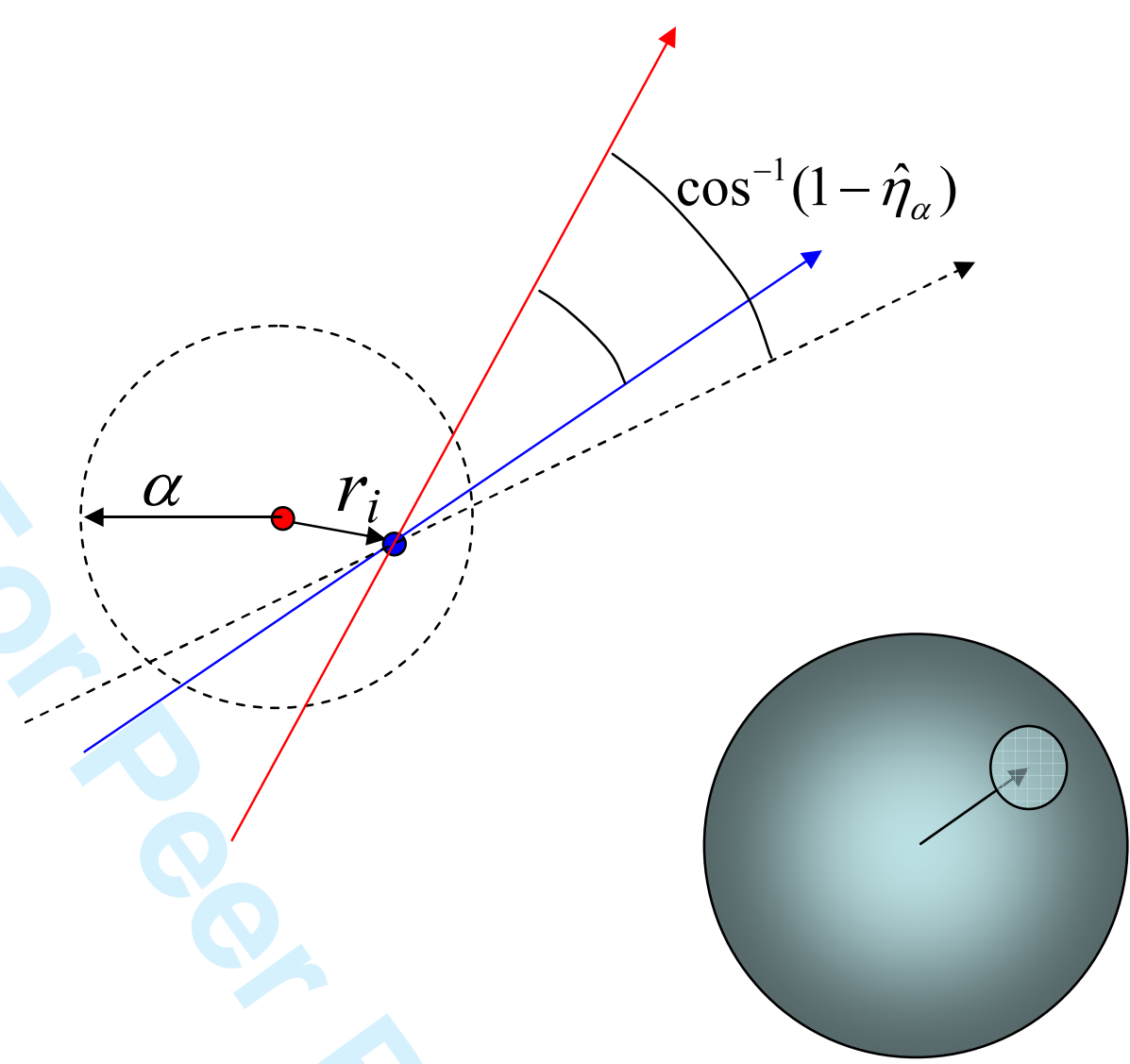

Figure 4. Depiction of some terms used to describe the relative positions of the $i$ th rigid linear molecule and its replicated partner. The red and blue dots are the centres of the original and replicated partner dumbbells respectively (after subtracting the replication vector $\mathbf{L}_{\mathbf{x}}$ from the position of the replicated dumbbell), while the red and blue lines are the directors of the original and replicated partner dumbbells respectively. $r_{\mathrm{i}}$, the relative separation of their centres is constrained to be less than $\alpha$, while $\tau_{i}$, the angle between their directors, is constrained to be less than $\cos ^{-1}\left(1-\hat{\eta}_{\alpha}\right)$. The relative azimuthal angle is not constrained, so the director of the replicated molecule can point anywhere within a circle on a sphere centred on the director or the original molecule. 


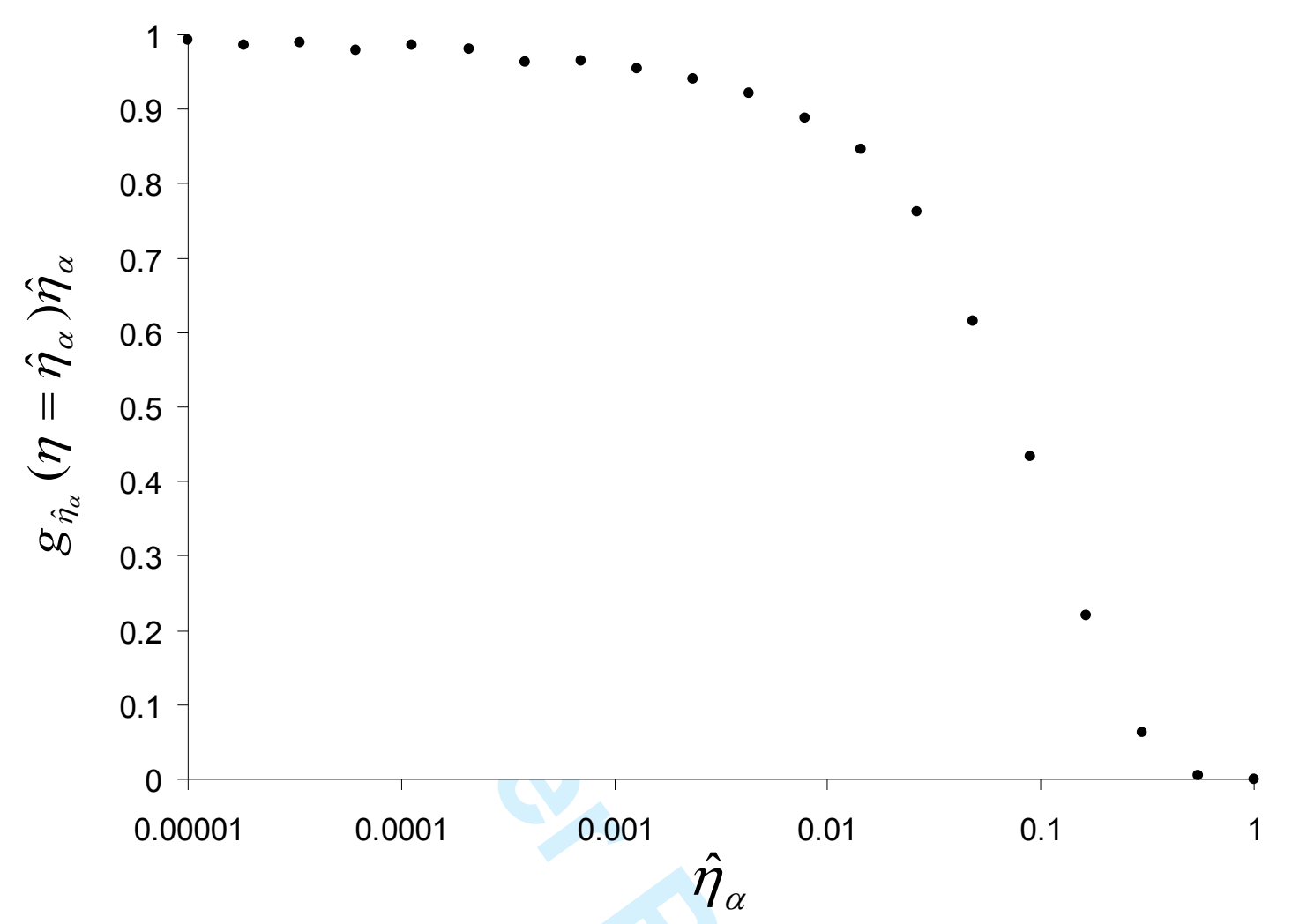

Figure 5. The variation of $g_{\hat{\eta}_{\alpha}}$ with $\hat{\eta}_{\alpha}$ for a hard face centred cubic hard dumbbell crystal with reduced bond length $L^{*}=L / \sigma=0.3$ and reduced molecular density $\rho^{*}=\rho \sigma^{3}=0.883397$, where $\sigma$ is the diameter of one site of the dumbbell. 


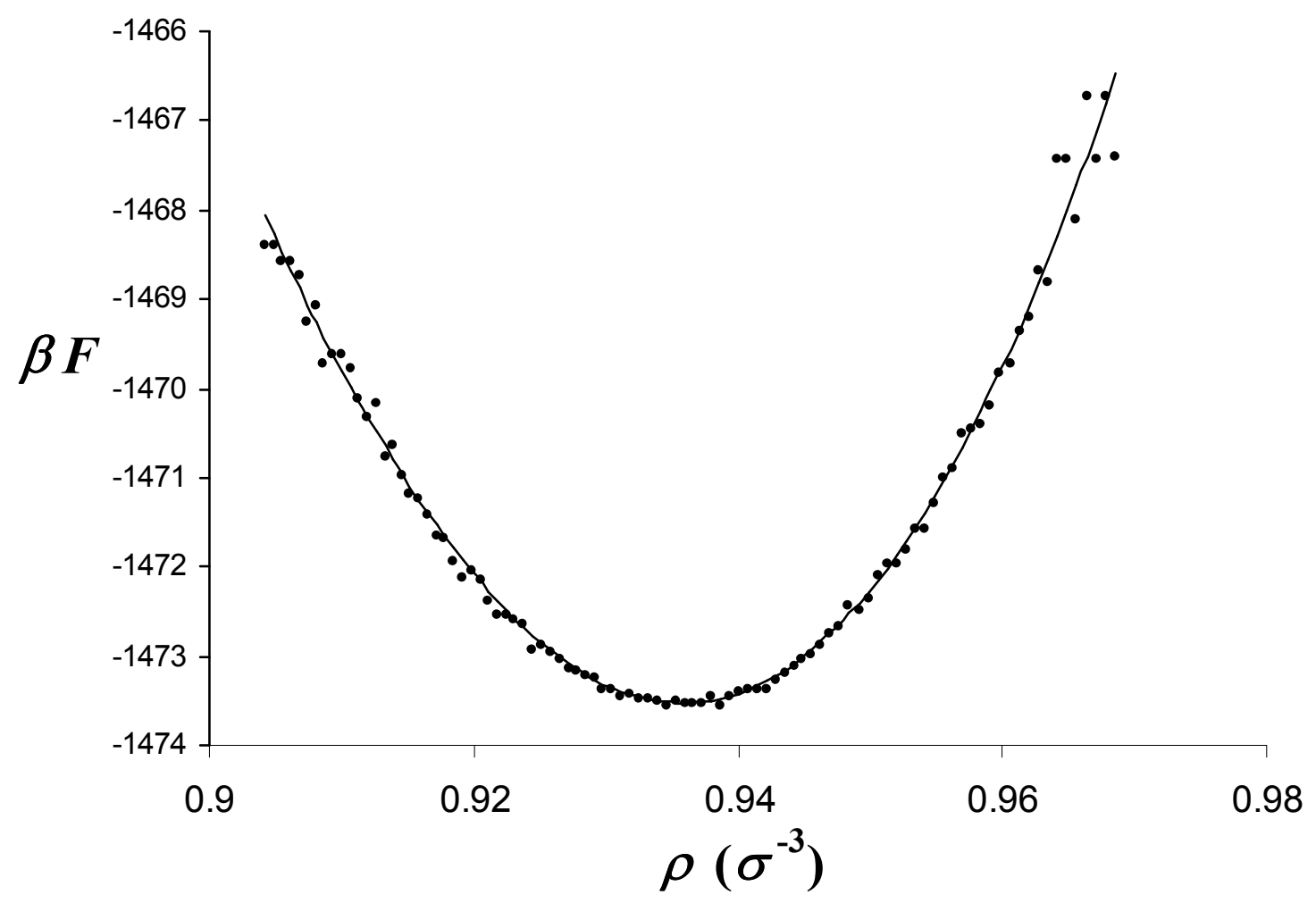

Figure 6. Reduced canonical Helmholtz free energy for the crystalline solid phase of a shifted-force Lennard-Jones system with 256 particles for a range of densities $\rho$ at reduced temperature $T^{*}=(\beta \varepsilon)^{-1}$ $=0.56$, reduced pressure $P^{*}=P \sigma^{3} / \varepsilon=0.00186$, and reduced configurational chemical potential $\mu^{*}=$ $\mu / \varepsilon=-3.23$, where $\sigma$ and $\varepsilon$ are the Lennard-Jones length and energy parameters respectively. The symbols are simulation data, while the line is a cubic polynomial fit to this data and is used to generate the free energy model for use in the indirect Gibbs simulation described in the text. 


\section{References}

M.B. Sweatman, A.A. Atamas, and J.M. Leyssale. The self-referential method for linear rigid bodies: application to hard and Lennard-Jones dumbbells. Journal of Chemical Physics, in print (2008).

M.B. Sweatman, A.A. Atamas, and J.M. Leyssale. The self-referential method combined with thermodynamic integration. Journal of Chemical Physics, 128, 064102 (2008).

M.B. Sweatman. Self-referential Monte Carlo method for calculating the free energy of crystalline solids. Physical Review E, 72, 016711 (2005).

M.B. Sweatman and N. Quirke. Simulating fluid-solid equilibrium with the Gibbs ensemble. Molecular Simulation, 30, 23 (2004).

C. Vega, E. Sanz, J.L.F. Abascal, and E.G. Noya. Determination of phase diagrams via computer simulation: methodology and applications to water, electrolytes and proteins. Journal of PhysicsCondensed Matter, 20, (2008).

B. Smit and D. Frenkel, Understanding molecular simulation: from algorithms to applications. (Academic, New York, 1996).

A.Z. Panagiotopoulos. Direct Determination of Phase Coexistence Properties of Fluids by Monte-Carlo Simulation in a New Ensemble. Molecular Physics, 61, 813 (1987).

A.Z. Panagiotopoulos, N. Quirke, M. Stapleton, and D.J. Tildesley. Phase-Equilibria by Simulation in the Gibbs Ensemble - Alternative Derivation, Generalization and Application to Mixture and Membrane Equilibria. Molecular Physics, 63, 527 (1988).

M. Mezei. Grand-canonical ensemble Monte-Carlo study of dense liquid Lennard-Jones soft-spheres and water. Molecular Physics, 61, 565 (1987).

P. Tilwani, Masters thesis, Colorado School of Mines, 2000.

K.G. Ayappa and C. Ghatak. The structure of frozen phases in slit nanopores: A grand canonical Monte Carlo study. Journal of Chemical Physics, 117, 5373 (2002).

B. Coasne, S.K. Jain, L. Naamar, and K.E. Gubbins. Freezing of argon in ordered and disordered porous carbon. Physical Review B, 76, (2007).

C. Alba-Simionesco, B. Coasne, G. Dosseh, G. Dudziak, K.E. Gubbins, R. Radhakrishnan, and M. Sliwinska-Bartkowiak. Effects of confinement on freezing and melting. Journal of Physics-Condensed Matter, 18, R15 (2006). 
B. Coasne, J. Czwartos, K.E. Gubbins, F.R. Hung, and M. Sliwinska-Bartkowiak. Freezing of mixtures confined in a slit nanopore. Adsorption-Journal of the International Adsorption Society, 11, 301 (2005).

J. Czwartos, B. Coasne, K.E. Gubbins, F.R. Hung, and M. Sliwinska-Bartkowiak. Freezing and melting of azeotropic mixtures confined in nanopores: experiment and molecular simulation. Molecular Physics, 103, 3103 (2005).

F.R. Hung, B. Coasne, E.E. Santiso, K.E. Gubbins, F.R. Siperstein, and M. Sliwinska-Bartkowiak. Molecular modeling of freezing of simple fluids confined within carbon nanotubes. Journal of Chemical Physics, 122, (2005).

F.R. Hung, K.E. Gubbins, R. Radhakrishnan, K. Szostak, F. Beguin, G. Dudziak, and M. SliwinskaBartkowiak. Freezing/melting of Lennard-Jones fluids in carbon nanotubes. Applied Physics Letters, 86, (2005).

B. Coasne, J. Czwartos, K.E. Gubbins, F.R. Hung, and M. Sliwinska-Bartkowiak. Freezing and melting of binary mixtures confined in a nanopore. Molecular Physics, 102, 2149 (2004).

F.R. Hung, G. Dudziak, M. Sliwinska-Bartkowiak, and K.E. Gubbins. Freezing/melting behaviour within carbon nanotubes. Molecular Physics, 102, 223 (2004).

R. Radhakrishnan, K.E. Gubbins, and M. Sliwinska-Bartkowiak. Global phase diagrams for freezing in porous media. Journal of Chemical Physics, 116, 1147 (2002).

M. Sliwinska-Bartkowiak, R. Radhakrishnan, and K.E. Gubbins. Effect of confinement on melting in slit-shaped pores: Experimental and simulation study of aniline in activated carbon fibers. Molecular Simulation, 27, 323 (2001).

M. Sliwinska-Bartkowiak, G. Dudziak, R. Gras, R. Sikorski, R. Radhakrishnan, and K.E. Gubbins. Freezing behavior in porous glasses and MCM-41. Colloids and Surfaces a-Physicochemical and Engineering Aspects, 187, 523 (2001).

M. Sliwinska-Bartkowiak, G. Dudziak, R. Sikorski, R. Gras, K.E. Gubbins, R. Radhakrishnan, and K. Kaneko. Freezing behavior in porous materials: Theory and experiments. Polish Journal of Chemistry, 75, 547 (2001).

M. Sliwinska-Bartkowiak, G. Dudziak, R. Sikorski, R. Gras, K.E. Gubbins, and R. Radhakrishnan. Dielectric studies of freezing behavior in porous materials: Water and methanol in activated carbon fibres. Physical Chemistry Chemical Physics, 3, 1179 (2001). 
M. Miyahara, H. Kanda, K. Higashitani, and K.E. Gubbins. Molecular simulation study on freezing in nano-pores. Characterization of Porous Solids V, 128, 31 (2000). M. Sliwinska-Bartkowiak, J. Gras, R. Sikorski, G. Dudziak, R. Radhakrishnan, and K.E. Gubbins. Experimental and simulation studies of melting and freezing in porous glasses. Characterization of Porous Solids V, 128, 141 (2000).

M. Sliwinska-Bartkowiak, G. Dudziak, R. Sikorski, R. Gras, R. Radhakrishnan, and K.E. Gubbins. Melting/freezing behavior of a fluid confined in porous glasses and MCM-41: Dielectric spectroscopy and molecular simulation. Journal of Chemical Physics, 114, 950 (2001).

R. Radhakrishnan, K.E. Gubbins, and M. Sliwinska-Bartkowiak. Effect of the fluid-wall interaction on freezing of confined fluids: Toward the development of a global phase diagram. Journal of Chemical Physics, 112, 11048 (2000). Carlo method. Physical Review Letters, 79, 3002 (1997).

J. Chang and S.I. Sandler. Determination of liquid-solid transition using histogram reweighting method and expanded ensemble simulations. Journal of Chemical Physics, 118, 8390 (2003). 
D. Frenkel and A.J.C. Ladd. New Monte-Carlo Method to Compute the Free-Energy of Arbitrary Solids - Application to the Fcc and Hep Phases of Hard-Spheres. Journal of Chemical Physics, 81, 3188 (1984).

G. Grochola. Constrained fluid lambda-integration: Constructing a reversible thermodynamic path between the solid and liquid state. Journal of Chemical Physics, 120, 2122 (2004).

C. Vega and E.G. Noya. Revisiting the Frenkel-Ladd method to compute the free energy of solids: The Einstein molecule approach. Journal of Chemical Physics, 127, (2007).

E. de Miguel, R.G. Marguta, and E.M. del Rio. System-size dependence of the free energy of crystalline solids. J Chem Phys, 127, 154512 (2007).

J.M. Polson, E. Trizac, S. Pronk, and D. Frenkel. Finite-size corrections to the free energies of crystalline solids. Journal of Chemical Physics, 112, 5339 (2000).

P.A. Monson and D.A. Kofke. Solid-fluid equilibrium: Insights from simple molecular models. Advances in Chemical Physics, Volume 115, 115, 113 (2000).

J.M. Rickman and R. LeSar. Free-energy calculations in materials research. Annual Review of Materials Research, 32, 195 (2002).

C.D. Barnes and D.A. Kofke. Self-referential method for calculation of the free energy of crystals by Monte Carlo simulation. Physical Review E, 65, 036709 (2002).

N.B. Wilding and A.D. Bruce. Freezing by Monte Carlo phase switch. Physical Review Letters, 85, $5138(2000)$.

N.B. Wilding. A new simulation approach to the freezing transition. Computer Physics Communications, 146, 99 (2002).

C. Vega, E.P.A. Paras, and P.A. Monson. On the Stability of the Plastic Crystal Phase of Hard Dumbbell Solids. Journal of Chemical Physics, 97, 8543 (1992).

C. Vega, E.P.A. Paras, and P.A. Monson. Solid-Fluid Equilibria for Hard Dumbbells Via Monte-Carlo Simulation. Journal of Chemical Physics, 96, 9060 (1992).

A.L. Galbraith and C.K. Hall. Solid-liquid phase equilibria for mixtures containing diatomic LennardJones molecules. Fluid Phase Equilibria, 262, 1 (2007).

A.Z. Panagiotopoulos. Monte Carlo methods for phase equilibria of fluids. Journal of PhysicsCondensed Matter, 12, R25 (2000). 

M. Mehta and D.A. Kofke. Implementation of the Gibbs Ensemble Using a Thermodynamic Model for One of the Coexisting Phases. Molecular Physics, 79, 39 (1993). J.R. Errington, P.G. Debenedetti, and S. Torquato. Quantification of order in the Lennard-Jones system. Journal of Chemical Physics, 118, 2256 (2003).

B. Chen, J.I. Siepmann, and M.L. Klein. Direct Gibbs ensemble Monte-Carlo simulations for solidvapour equilibria: applications to Lennard-Jonesium and carbon dioxide. Journal of Physical Chemistry B, 105, 9840 (2001). 